IRSTI $29.27 .39 ; 29.27 .47$

https://doi.org/10.26577/phst-2019-2-p4

\title{
Generation of ultrahigh fields by microbubble implosion
}

\author{
M. Murakami ${ }^{*}$, A. Arefiev ${ }^{2}$, M.A. Zosa ${ }^{1}$ and J. Honrubia ${ }^{3}$ \\ ${ }^{1}$ Institute of Laser Engineering, Osaka University, Osaka, 565-0871, Japan \\ ${ }^{2}$ UC San Diego, 9500 Gilman Drive, 92093-0411, La Jolla, California, USA \\ ${ }^{3}$ School of Aeronautical and Space Engineering, Madrid Polytechnic University, \\ 3, Plaza adrenal Cisneros, Madrid, Spain \\ *e-mail:murakami-m@ile.osaka-u.ac.jp
}

\begin{abstract}
Breaking the 100-MeV barrier for proton acceleration will help elucidate fundamental physics and advance practical applications from inertial confinement fusion to tumor therapy. A novel concept of "microbubble implosion (MBI)" is proposed. In the MBI concept, bubble implosion combines micro-bubbles and ultraintense laser pulses of $10^{20}-10^{22} \mathrm{Wcm}^{-2}$ to generate ultrahigh fields and relativistic protons. The bubble wall protons are subject to volumetric acceleration toward the center due to the spherically symmetric electrostatic force generated by hot electrons filling the bubble. Such an implosion can generate an ultrahigh density proton core of nanometer size on the collapse, which results in an ultrahigh electrostatic field to emit energetic protons in the relativistic regime. Laser intensity scaling is investigated for accelerated proton energy and attainable electrostatic field using MBI. Three-dimensional particle-in-cell and molecular dynamics simulations are conducted in a complementary manner. As a result, underlying physics of MBI are revealed such as bubble-pulsation and ultrahigh energy densities, which are higher by orders of magnitude than, for example, those expected in a fusion-igniting core of inertially confined plasma. MBI has potential as a plasma-optical device, which optimally amplifies an applied laser intensity by a factor of two orders of magnitude; thus, MBI is proposed to be a novel approach to the Schwinger limit.
\end{abstract}

Key words: Ultraintense ultrashort laser, relativistic protons, Schwinger limit, microbubble implosion, high-energydensity physics.

PACS numbers: 52.38.Kd, 52.30.Ex, 52.40.Kh, 52.65.-y

\section{Introduction}

In the past quarter century, the chirped-pulseamplification (CPA) technique has increased the laser intensity more than ten mil-lion times [1]. Consequently, diverse research via laser-matter interactions has been pursued. Examples include fast ignition [2-4] and high energy particle acceleration for electrons and ions with respect to different applications [5-8]. These studies have been conducted under the relativistic electron regime [9], corresponding to the laser intensity $\mathrm{I}_{\mathrm{L}}$ with $10^{18}<\mathrm{I}_{\mathrm{L}}$ $\left(\mathrm{Wcm}^{-2}\right)<10^{22}$. Utilizing laser fields is, however, far from straightforward because of their rapid oscillations. This presents a fundamental difficulty, particularly when attempting to accelerate ions that can then be used for a range of promising applications. A common approach to circumvent this difficulty is to first heat electrons in a laser-irradiated target [10-14].

As the heated electrons expand, they generate a strong electric field at the target surface that causes ions to accelerate [15-18]. For example, Coulomb explosion is a well-known scheme for ion acceleration [19-22], in which $\mathrm{nm}$ - to $\mu \mathrm{m}$-sized clusters are irradiated by an intense laser pulse to blow off most of the electrons in a moment. The remaining ions then begin to spherically expand due to the strong Coulomb force. All these ion acceleration schemes strongly depend on the laser intensity applied on the targets. As well as the particle acceleration, high field science such as vacuum physics [23-29] is also important and fundamental topics in high energy density physics via laser. In quantum electrodynamics (QED), the Schwinger limit, $E_{\mathrm{S}}=m_{\mathrm{e}}^{2} c^{3} / e h \sim 1.3 \times 10^{18} \mathrm{Vm}^{-1}$, is reported as the scale above which the electromagnetic field is expected to create electron-positron pairs spontaneously, where $m_{\mathrm{e}}$ is the electron mass, $c$ is the speed of light in a vacuum, $e$ is the elementary charge, and $h$ is the Planck constant. The electric field at the Schwinger limit corresponds to the laser intensity $\mathrm{I}_{\mathrm{L}} \sim 2.3 \times 10^{29} \mathrm{Wcm}^{-2}$. To date, many studies have focused on increasing laser performance with 
regard to power and intensity [30-33]. While the threshold for electron-positron pair production may be substantially below $E_{S}$ for multiple focusing pulses $[34,35]$, the current "distance" in the laser intensity to the Schwinger limit is still roughly five to six orders of magnitude away even by taking such measures into account.

Recently we have proposed a conceptually new approach to generate ultrahigh fields and resultant high energy protons, which is referred to as "microbubble implosion" (MBI), picture is illustrated in Figure 1(a). Suppose that a spherical micron-sized bubble, prepared artificially in a solid target, is forced to implode when placed into a heat bath composed of hot electrons (Figure 1(b)), which are actively circulating in and outside of the bubble [36]. For simplicity, the target is assumed to be pure hydrogen.
The ion implosion continues until the ions become compressed to a nanometer scale such that their radial inward motion is halted by the resulting outward electric field. This means further compression of the original laser energy in space and time in the shape of an extremely dense ion core, which leads to generation of an ultrahigh electric field at the center. This field is much stronger than the field that initiated the implosion, and it causes a violent explosion of the compressed ions, with resulting energies many times higher than the energy gained during the implosion. Below we construct a simple semi-analytical model, which encapsulates the important features obtained from multidimensional simulations. Not only can this model easily visualize the underlying physics of this novel phenomenon but also define the limiting performance.
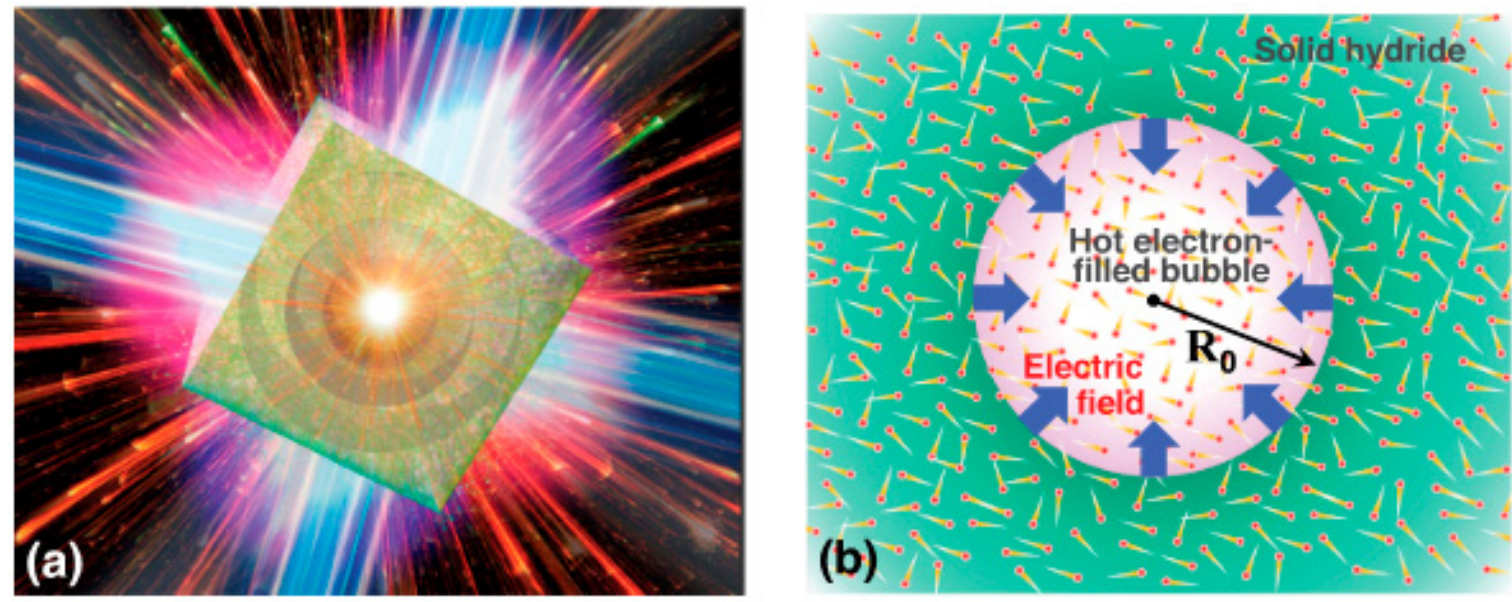

Figure 1 - (a) Envisioned picture showing all of the main events of microbubble implosion (MBI), i.e., laser illumination, hot electron spread, implosion, and proton flash at the end.

(b) Schematic picture showing the core mechanism of MBI. Being filled with hot electrons in the bubble, the bulk of the protons begin to accelerate toward the center in a spherically symmetric manner.

Phenomena such as converging shock waves and sonoluminescence are similar to a bubble implosion. Shock waves are observed in many branches of physics. Although sonoluminescence is a relatively new phenomenon in the acoustics field, Lord Rayleigh proposed the basic idea (contraction of a water bubble) over a century ago. The behavior of bubble implosions reported in this study remarkably differs. Extremely high temperatures and low densities characterize the physical states of collapsing converging waves at the center in shock waves and sonoluminescence. By contrast, extremely high densities and practically zero temperatures for protons characterize bubble implosions.

\section{Dynamics of Microbubble Implosion}

\subsection{One-dimensional hybrid simulation}

Hot electron distribution in the bubble is the key physical issue in MBI. We first conducted 1D hybrid simulations, in which electrons and ions are treated as an electrostatic field and particles, respectively. Practically, the specific value of the ionization degree in space, $\mathrm{a}=n_{\mathrm{i} 0} / n_{\mathrm{s} 0}$, results from the interplay between the laser and the target material. Hence, it depends on the external parameters such as the absorbed laser energy and the target volume. The electron distribution in the bubble is obtained by solving the Poisson Boltzmann (P-B) equation, $\nabla \varphi=4 \pi e\left[n_{\mathrm{ec}} \exp \left(e \varphi / T_{\mathrm{e}}\right)\right.$ 
$\left.-n_{\mathrm{i}}\right]$, where $\varphi$ is the electric potential and $n_{\mathrm{ec}}$ is the temporal electron density at the center. After normalizing the $\mathrm{P}-\mathrm{B}$ equation, the present system is found to depend on a single dimensionless parameter defined by $\Lambda=R_{0} / \lambda_{\mathrm{Di}}$, where $\lambda_{\mathrm{Di}}=\left(T_{\mathrm{e}} / 4 \pi n_{\mathrm{i} 0} e^{2}\right)^{1 / 2}$ is the Debye length. As a function of $\Lambda$, the P-B equation is numerically solved to give $\varphi(r)$ and consequently $\mathrm{n}_{\mathrm{e}}(\mathrm{r})$ under the appropriate boundary conditions. The potential profile $\varphi(r)$ evolves over time in accordance with the temporal ion density profile $n_{\mathrm{i}}(r)$. The two dimensionless variables, $R_{1} / R_{0}$ and $\Lambda$, constitute the analysis as control parameters.
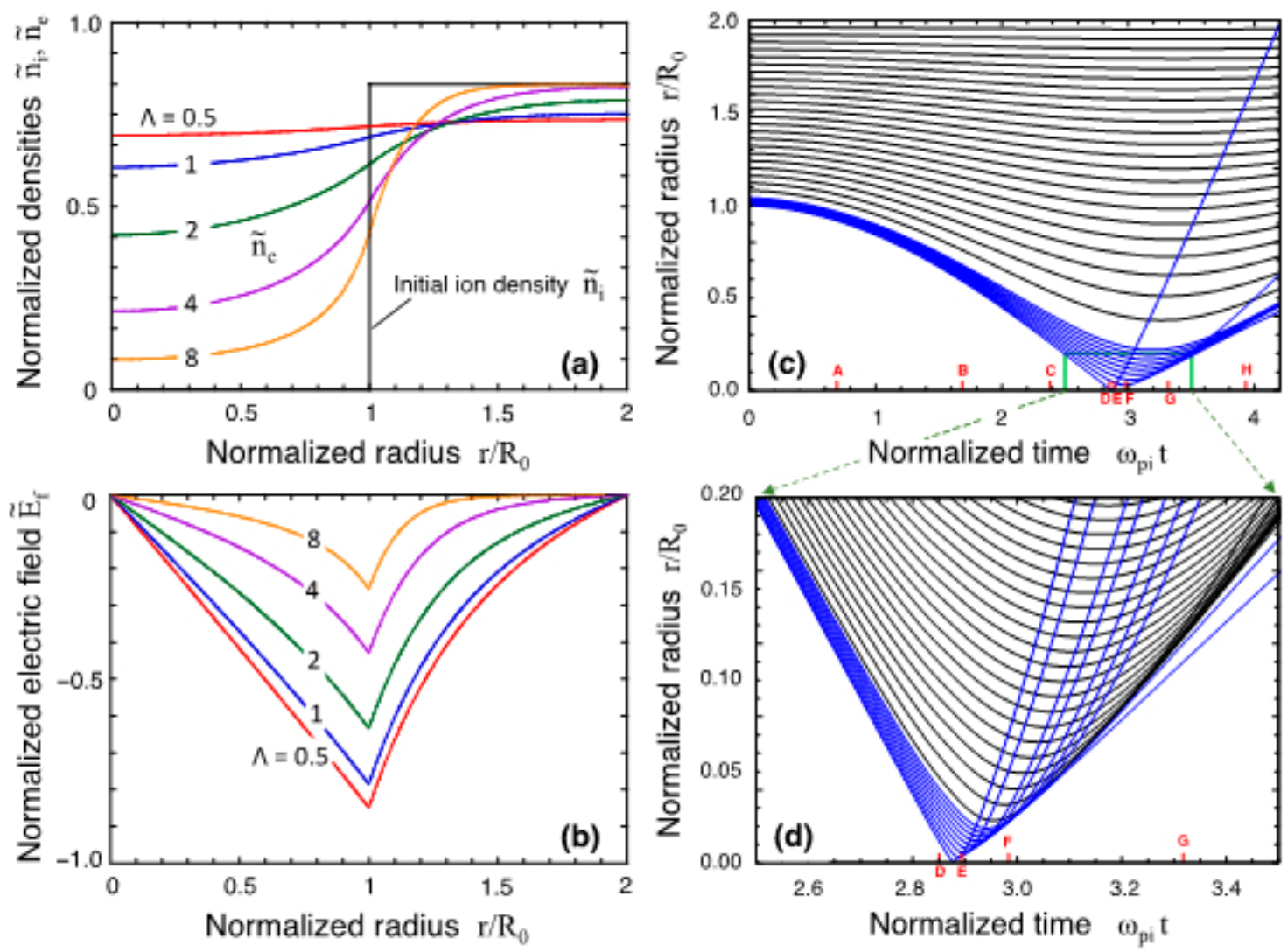

Figure 2 - (a) Initial density profiles of the electron and the ion normalized by the initial ion density, respectively. (b) the electric field as a function of $\Lambda$. (c) Ion trajectories obtained by $1 \mathrm{D}$ simulation under $R_{1} / R_{0}=2$ and $\Lambda=0.5$. The black curves correspond to initial radii, while the blue curves divide the innermost segment to better observe the implosion dynamics. The labels, $\mathrm{A}-\mathrm{H}$, are to compare other physical quantities in the following Figures. For the solid density, the normalized unit time corresponds to $3.4 \mathrm{fs}$. (d) Magnified view of the rectangle part in (c).

Figures 2(a) and (b) show the initial profiles for the electron density $n_{\mathrm{e}}(r)$ and the electric field, respectively, obtained for different values of $\Lambda$ and a fixed initial ion density profile normalized by $n_{\mathrm{i} 0}$. The electron profiles for $\Lambda<1$ are rather flat over the entire domain, while they conspicuously reduce in the bubble with increasing $\Lambda(>2)$. It is convenient to normalize time $t$ and use the dimensionless quantity $\omega_{p i 0} t$ instead, where $\omega_{p i 0}=\sqrt{4 \pi n_{i 0} Z^{2} e^{2} / m_{i}}$ is the ion plasma frequency and $m_{\mathrm{i}}$ is the ion mass.

Figure 2(c) shows the ion trajectories for the entire time region under the bubble conditions of
$R_{1} / R_{0}=2$ and $\Lambda=0.5$. The black curves correspond to initial radii with a constant increment of $\Delta r=$ $0.04 R_{0}$, while the blue curves subdivide the innermost segment to better resolve the implosion dynamics. The labels along the time axis, $\mathrm{A}-\mathrm{H}$, are to compare other physical quantities in subsequent figures.

Figure 2(d) shows a zoom-in of the rectangular in Figure 2(c). Until time D, all of the ion trajectories remain laminar, so that one curve does not intersect another. However, upon the collapse (time E), the innermost trajectory is strongly ejected radially outwards and this is the phenomenon that we call the proton flash. In Figure 2(d), the innermost seven 
trajectories in blue represent flashed protons and they behave quite differently from the other trajectories. These trajectories sharply cut across the other trajectories, confirming that the flashed protons quickly slide down a Coulomb potential that can be effectively viewed as quasi-static. These "runaway" protons are emitted from a very small volume with $r$ $\sim 0.05 R_{0}$ due to an explosive acceleration under the ultrahigh electric field that is generated by the accumulated proton core at the center.
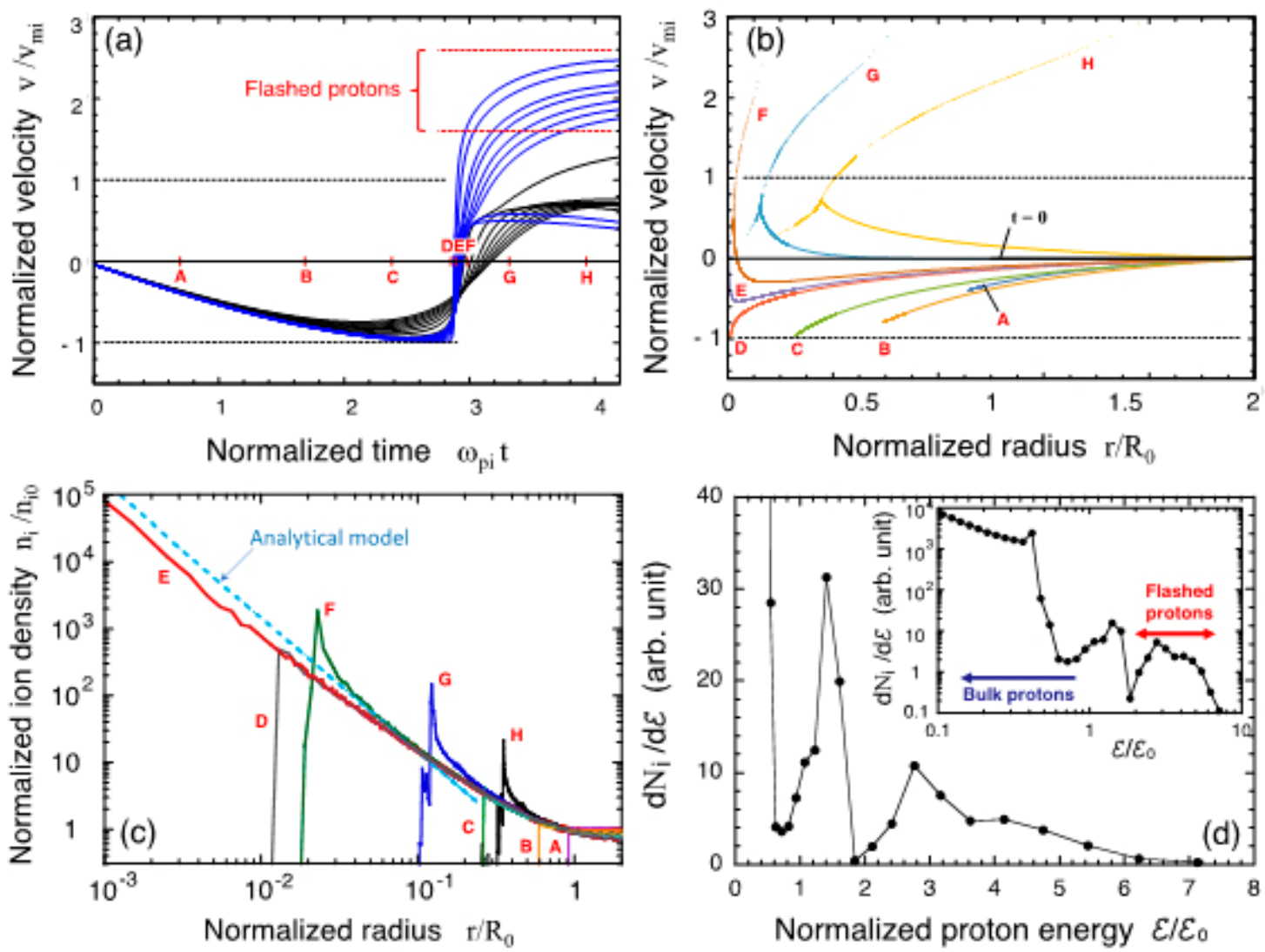

Figure 3 - (a) Temporal evolution of the velocities of individual protons normalized by the maximum implosion velocity $v_{\mathrm{mi}}$. (b) Overall view of the velocity profiles at the snapshot times $\mathrm{A}-\mathrm{H}$.

(c) Density profiles normalized by the solid density at different times A - H. (d) Energy spectrum at time H. The inset stands for the same data given in the main frame but in double-logarithmic scales.

The unit of the energy is chosen to be the maximum kinetic energy in the implosion phase. The two-humped structure is attributed to the acceleration process at the singular behavior at the center

Figure 3(a) shows the velocity evolution of the flashed protons and the surrounding protons, normalized by the maximum implosion velocity $v_{\mathrm{mi}}$. The blue and black curves correspond to those in Figure 2(d). Upon the collapse (times D - F), the velocity of the flashed protons drastically increases, exceeding the maximum implosion velocity by a factor of $2.0-2.5$, which are simply squared to give corresponding energy amplification by a factor of 4 - 6. This energy amplification for the flashed protons is due to their sliding down the steep Coulomb potential slope. The innermost protons are the first ones to be reflected near the center. The dynamics of the other protons that follow and that are located a bit outwards is similar, but the expulsion is slightly delayed and the resulting energy amplification factor is smaller.

Figure 3(b) shows snapshots of the proton velocity as a function of radius for times $\mathrm{A}-\mathrm{H}$ in Fig. 2(c). For practical laser and target parameters, the proton flash occurs over a very short time interval and a very small volume corresponding to times $\mathrm{D}-\mathrm{F}$. The flashed protons have a much higher velocity than surrounding bulk protons, as can be seen in snapshots 
$\mathrm{G}$ and $\mathrm{H}$. It should be noted that, at such later times, a snowplow-like two-stream-structure is formed.

\subsection{Three-dimensional Particle-In-Cell (PIC)} simulation

We performed 3D PIC simulations with an opensource code EPOCH [37]. We employed such a periodic boundary condition that a single bubble was located at the center of a cubic plasma volume with one side of $L=1200 \mathrm{~nm}$ and the minimum cell size of $6 \mathrm{~nm}$. For simplicity, laser-matter interaction was not taken into account. Initially, the bubble of $R_{0}=$ $300 \mathrm{~nm}$ was set to be empty, and a uniform chargeneutral plasma surrounding the bubble was composed of hot electrons with $T_{\mathrm{e}}=10 \mathrm{MeV}$ and cold ions with density of $n_{\mathrm{i} 0}=n_{\mathrm{e} 0}=10^{21} \mathrm{~cm}^{-3}$.
Figure 4(a) shows the snapshots of bubble implosion with the proton density distributions colorcoded. The pulsating behavior of MBI is robust despite that the shrinking bubble is substantially deformed into a squared shape due to accumulated numerical errors under the Cartesian mesh employed in the PIC code. Figure 4(b) shows the temporal evolution of the proton energy spectrum. The pulsation period is estimated to be $T_{\text {cyc }} \sim 150 \mathrm{fs}$, which indeed agrees with the interval between the $1 \mathrm{st}$ $(t \sim 70 \mathrm{fs})$ and the 2nd ( $t \sim 220 \mathrm{fs})$ proton flash. Furthermore, throughout the explosion phase, the maximum implosion energy of $230 \mathrm{keV}$ is amplified up to $1.4 \mathrm{MeV}$ with an amplification factor of $\sim 6$, which agrees well with the results observed in the 1D hybrid simulation.
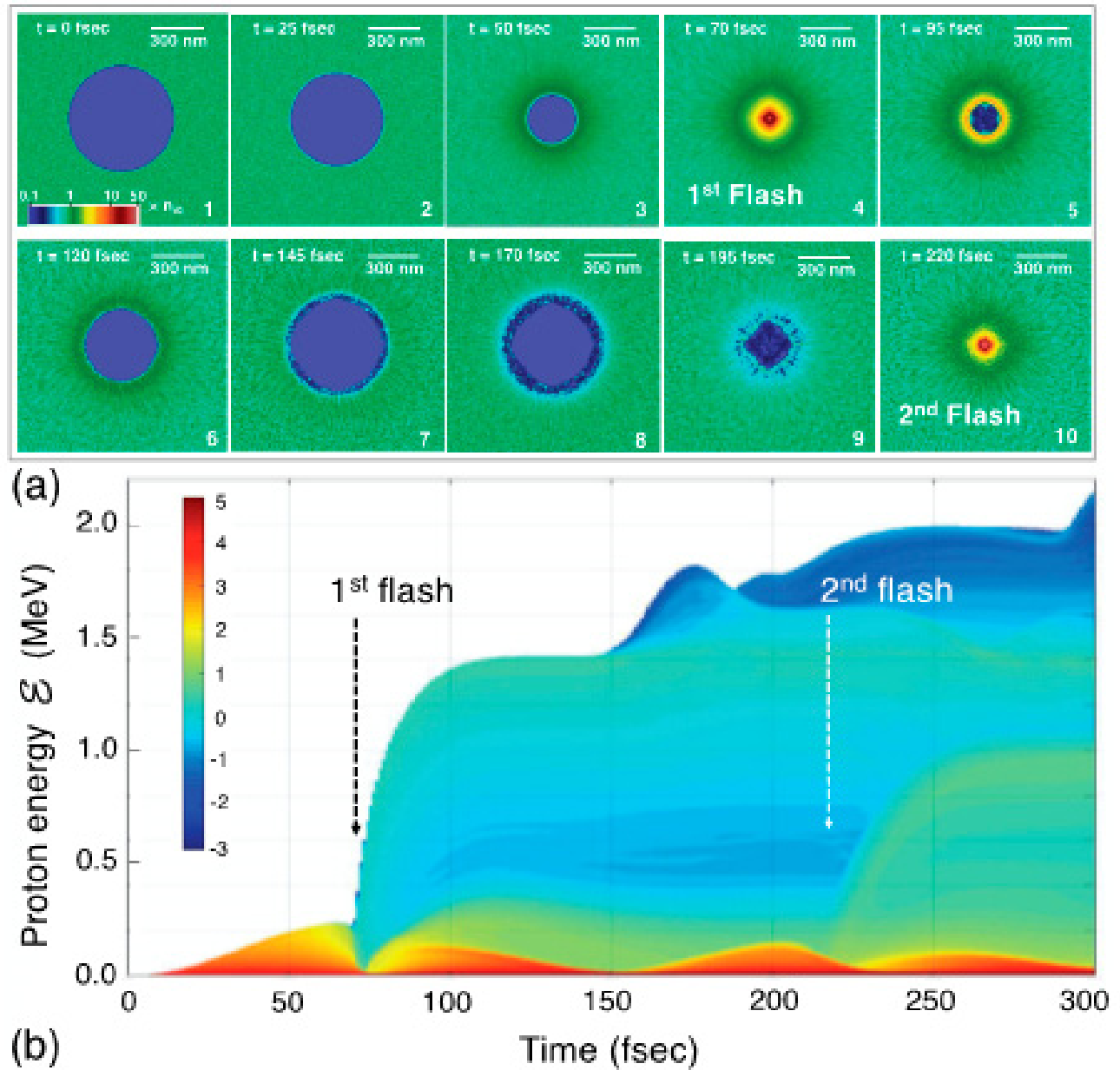

Figure 4 - 3D PIC simulation of MBI. Initially, the bubble of $R_{0}=300 \mathrm{~nm}$ is empty.

A uniform charge-neutral plasma surrounding the bubble is composed of hot electrons with $T_{\mathrm{e}}=10 \mathrm{MeV}$ and cold protons with density of $n_{\mathrm{i} 0}=n_{\mathrm{e} 0}=10^{21} \mathrm{~cm}^{-3}$. (a) Snapshots of the bubble collapse with the proton density distributions being color-coded. (b) Temporal evolution of the proton energy spectrum for the same case as in panel (a). The integers $\beta$ assigned along the color bar in panel (b) indicate proton density ni such that $n_{\mathrm{i}}=10^{\beta} \mathrm{cm}^{-3} \mathrm{eV}^{-1}$. 


\section{Generation of Ultrahigh-Energy-Density Nanosphere}

While PIC simulations can provide a comprehensive physical picture by treating a lot of particles, the dynamic range is limited because of the fixed size of the cartesian cells. In contrast, molecular dynamic (MD) simulations can treat the dynamics over a much wider dynamic range, taking all binary collisions into account, but because of that they are limited to a much smaller number of particles.

Figure 5(a) compares the density profiles at the maximum compression obtained by 3D MD simulations and the 1D model. The fixed parameters are $R_{0}=1 \mathrm{~nm}$ and $n_{\mathrm{i} 0}=5 \times 10^{22} \mathrm{~cm}^{-3}$, assuming $\Lambda<<1$.
The inset shows the initial configuration of the pseudo-particles used for the simulations, where we took the innermost four atomic layers into account. One thousand pseudo-protons are uniformly arranged on each of the four layered spherical surfaces [38] with an interatomic distance $d_{0}=0.27$ $\mathrm{nm}$. Each pseudo-proton carries a mass and charge corresponding to about 10 real protons. The electrons were treated as a uniform background. Here, it should be noted that in the 3D MD simulations, all binary collisions between the pseudo-protons are precisely computed. This feature is indispensable when evaluating proton dynamics on such an infinitesimally small scale as nanometer.
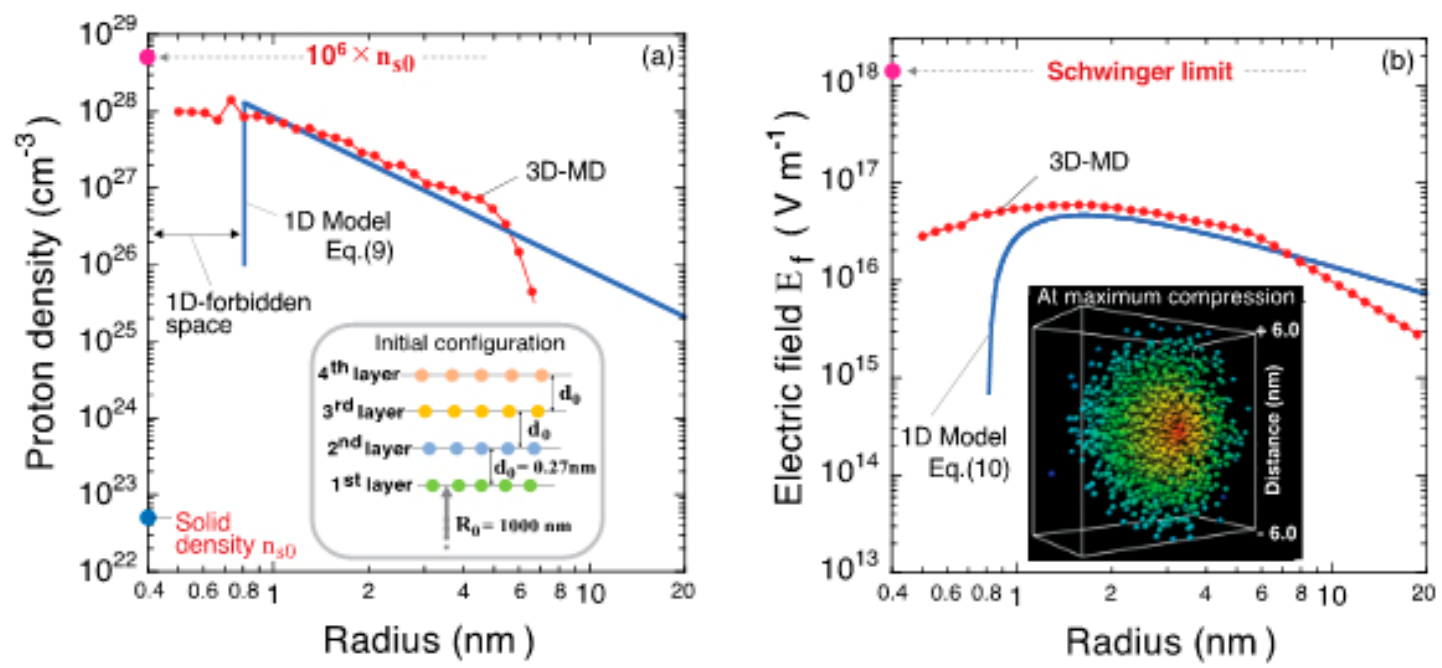

Figure 5 - (a) Comparison of the proton density at the maximum compression between

the 3D molecular dynamic simulations and the $1 \mathrm{D}$ model. Fixed parameters are $R_{0}=1 \mathrm{~nm}$ and $n_{\mathrm{i} 0}=5 \times 10^{22} \mathrm{~cm}^{-3}$. The curve labeled "3D-MD" is obtained using the innermost four layers as shown in the inset.

(b) Comparison of the electrostatic fields between the simulations and the model. The inset shows the proton distribution around the center (color-coded in accordance with the distance from the center).

It is remarkable that the four atomic layers eventually stagnate after imploding down to the small radii $<6 \mathrm{~nm}$. Upon the bubble collapse, the particles scatter around the 1D minimum radius, $r$ $=r_{\min } \sim 0.8 \mathrm{~nm}$, by random collisions, and a substantial part of them is further compressed into an even smaller central volume (1D-forbidden space). The characteristic time interval of proton stagnation at the center is about 10 as. The observed average proton densities inside the sphere for $r<1 \mathrm{~nm}$ are roughly $10^{28} \mathrm{~cm}^{-3}$ as demonstrated in Figure 5(a). Meanwhile, the maximum density predicted by the $1 \mathrm{D}$ model is
$1.3 \times 10^{28} \mathrm{~cm}^{-3}$, which together with the overall spatial profile agrees well with the simulation result.

Figure 5(b) compares the electric field obtained by the $1 \mathrm{D}$ model, with the 3D MD simulation. The 1D curve excellently reproduces the simulation curve in the core volume for $r<6 \mathrm{~nm}$. By contrast, for $\mathrm{r}>$ $6 \mathrm{~nm}$, the simulation curve decays more swiftly than the $1 \mathrm{D}$ curve according to the power law, $E_{\mathrm{f}} \sim r^{-2}$. This is because the almost all the protons are accumulated in the core volume at the maximum compression. The inset shows the hemispherical perspective view of the protons distributed around the target center at the 
maximum compression, where distances of the proton from the center are color-coded. To the best of our knowledge, we do not know of any other principles published in the literature to achieve such unprecedented physical quantities on earth as the compressed density on the order of $10^{5}-10^{6}$ times the solid density and the electrostatic field on the order of $10^{16}-10^{17} \mathrm{Vm}^{-1}$.

\section{Conclusions}

We propose a novel concept, bubble implosion, to generate an ultrahigh field to accelerate pro- tons to relativistic energies. A simple model and 1D, 2D, and 3D simulations comprehensively investigate the dynamics of the bubble implosion. This phenomenon is very likely to occur in reality. A stable implosion shrinks to a nanometer size and achieves an ultradense proton core, forming an unprecedentedly high electric field and producing proton flashes. The generation of an ultrahigh field is attributed to spherical convergence to the center. Moreover, Coulomb-imploded bubbles are robust and behave as nano-pulsars repeating implosion and explosion to emit energetic protons. Although the present paper assumes pure hydrogen targets, a modified scenario should be applicable to other hydrides.

Current laser technology is suitable to experimentally identify bubble implosion by observing proton emissions at relativistic energies, which will be a major breakthrough to crack the 100 $\mathrm{MeV}$ barrier. For such experiments, a uniform and well-activated Coulomb field must be created inside the bubbles by laser irradiation of micron-sized bubbles embedded inside a solid target. We have demonstrated in terms of the $2 \mathrm{D}$ simulation that a symmetric bubble implosion can be achievable even under a realistic condition of laser-matter interaction. Consequently, the present concept should provide a new platform to elucidate fundamental phenomena in the fields of high-energy-density physics [39] and astrophysics.

Microbubble implosion thus holds promise in principle to achieve such an ultrahigh-energy-density state of matter that is higher by orders of magnitude than those expected in a fusion-igniting core of inertially confined plasma. Introducing high- $Z$ materials to MBI as the target composition or a surface coating are expected to achieve even higher electric fields and resultant proton energies. MBI has potential as a plasma-optical device, which optimally amplifies the applied laser intensity by a factor of two orders of magnitude.

\section{Acknowledgments}

M.M. was supported by the Japan Society for the Promotion of Science (JSPS). A.A. was supported by the Air Force Office of Scientific Research under Award No. FA9550-17-1-0382.

\section{References}

1. Strickland D. \& Mourou G. (1985). Compression of amplified chirped optical pulses. Opt. Commun., 55, 447449.

2. Tabak M., Hammer I., Glinsky M. E., Kruer W. L., Wilks S. C., Woodworth J., Campbell E. M., Perry M. D., \& Mason R. J. (1994). Ignition and high gain with ultrapowerful lasers. Phys. Plasmas, 1, 1626.

3. Wilks S. C., Langdon A. B., Cowan T. E., Roth M., Singh M., Hatchett S., Key M. H., Pennington D., MacKinnon A., \& Snavely R. A. (2001). Energetic proton generation in ultra-intense laser-solid interactions. Phys. Plasmas, 8, 542.

4. Roth M., Cowan T. E., Key M. H., Hatchett S. P., Brown C., Fountain W., Johnson J., Pennington D. M., Snavely R. A., Wilks S. C., Yasuike K., Ruhl H., Pegoraro F., Bulanov S. V., Campbell E. M., Perry M. D., \& Powell H. (2001). Fast ignition by intense laser-accelerated proton beams. Phys. Rev. Lett., 86, 436-439.

5. Daido H., Nishiuchi M., \& Pirozhkov A. S. (2012). Review of laser-driven ion sources \& their applications. Rep. Prog. Phys., 75, 056401.

6. Tajima T. \& Dawson J. M. (1979). Laser Electron Accelerator. Phys. Rev. Lett., 43, 267.

7. Bulanov S. V. \& Khoroshkov V. S. (2002). Feasibility of using laser ion accelerators in proton therapy. Plasma Phys. Rep., 28, 453-456.

8. Gonsalves A. J., Nakamura K., Daniels J., Benedetti C., Pieronek C., de Raadt T. C. H., Steinke S., Bin J. H., Bulanov S. S., van Tilborg J., Geddes C. G. R., Schroeder C. B., Toth Cs., Esarey E., Swanson K., Fan-Chiang L., Bagdasarov G., Bobrova N., Gasilov V., Korn G., Sasorov P., \& Leemans W. P. (2019). Petawatt Laser Guiding and Electron Beam Acceleration to $8 \mathrm{GeV}$ in a Laser-Heated Capillary Discharge Waveguide. Phys. Rev. Lett., $122,084801$. 
9. Atzeni S. \& Meyer-ter-Vehn J. (2004). Fast ignition. In The Physics of Inertial Fusion: BeamPlasma Interaction, Hydrodynamics, Hot Dense Matter. Oxford University Press.

10. Malka V., Fritzler S., Lefebvre E., dfHumieres E., Ferrand R., Grillon G., Albaret C., Meyroneinc S., Chambaret J.-P., Antonetti A., \& Hulin D. (2004). Practicability of protontherapy using compact laser systems. Med. Phys., $31,1587$.

11. Borghesi M., Fuchs J., Bulanov S. V., Mackinnon A. J., Patel P. K., \& Roth M. (2006). Fast Ion Generation by High-Intensity Laser Irradiation of Solid Targets and Applications. Fusion Sci. Technol., 49, 412.

12. Nakamura T., Bulanov S. V., Esirkepov T., \& Kando M. (2010). High-Energy Ions from Near-Critical Density Plasmas via Magnetic Vortex Acceleration. Phys. Rev. Lett., 105, 135002.

13. Yin L., Albright B. J., Bowers K. J., Jung D., Fernandez J. C., and Hegelich B. M. (2011). Three-Dimensional Dynamics of Breakout Afterburner Ion Acceleration Using High-Contrast Short-Pulse Laser and Nanoscale Targets. Phys. Rev. Lett., 107, 045003.

14. Weng S. M., Liu M., Sheng Z. M., Murakami M., Chen M., Yu L. L., \& Zhang J. (2016). Dense blocks of energetic ions driven by multi-petawatt lasers. Sci. Rep., 6, 22150.

15. Esirkepov T., Borghesi M., Bulanov S. V., Mourou G., \& Tajima T. (2004). Highly Efficient Relativistic-Ion Generation in the Laser-Piston Regime. Phys. Rev. Lett., 92, 175003.

16. Murakami M. \& Basko M. M. (2006). Self-similar expansion of finite-size non-quasi-neutral plasmas into vacuum: Relation to the problem of ion acceleration. Phys. Plasmas, 13, 012105.

17. Bulanov S. S., Bychenkov V. Yu., Chvykov V., Kalinchenko G., Litzenberg D. W., Matsuoka1 T., Thomas A. G. R., Willingale L., Yanovsky V., Krushelnick K., \& Maksimchuk A. (2010). Generation of GeV protons from 1 PW laser interaction with near critical density targets. Phys. Plasmas, 17, 043105.

18. Haberberger D., Tochitsky S., Fiuza F., Gong C., Fonseca R. A., Silva L. O., Mori W. B., \& Joshi C. (2012). Collisionless shocks in laser-produced plasma generate monoenergetic high-energy proton beams. Nat. Phys., 8, 95-99.

19. Ditmire T., Zweiback J., Yanovsky V. P., Cowan T. E., Hays G., \& Wharton K. B. (1997). High-energy ions produced in explosions of superheated atomic clusters. Nature, 386, 54-56.

20. Zweiback J., Smith R. A., Cowan T. E., Hays G., Wharton K. B., Yanovsky V. P., \& Ditmire T. (2000). Nuclear Fusion Driven by Coulomb Explosions of Large Deuterium Clusters. Phys. Rev. Lett., 84, 2634.

21. Murakami M. \& Mima K. (2009). Efficient generation of quasimonoenergetic ions by Coulomb explosions of optimized nanostructured clusters. Phys. Plasmas, 16, 103108.

22. Peano F., Peinetti F., Mulas R., Coppa G., \& Silva L. O. (2006). Kinetics of the Collisionless Expansion of Spherical Nanoplasmas. Phys. Rev. Lett., 96, 175002.

23. Sauter F. (1931). Uber das Verhalten eines Elektrons im homogenen elektrischen Feld nach der relativistischen Theorie Diracs. Z. Phys., 69, 742.

24. Heisenberg W. \& Euler H. (1936). Consequences of Dirac's theory of positrons. Z. Phys. 98, 714.

25. Schwinger J. (1951). On gauge invariance and vacuum polarization. Phys. Rev., 82, 664.

26. Popov V. S. (2002). On Schwinger mechanism of $\mathrm{e}^{+} \mathrm{e}^{-}$pair production from vacuum by the field of optical and X-ray lasers. Phys. Lett. A, 298, 83.

27. Narozhny N. B., Bulanov S. S., Mur V. D., \& Popov V. S. (2004). On $\mathrm{e}^{+} \mathrm{e}^{-}$pair production by a focused laser pulse in vacuum. Phys. Lett. A, 330, 1.

28. DiPiazza A., Lotstedt E., Milstein A. I., \& Keitel C. H. (2009). Barrier control in tunneling $\mathrm{e}^{+}-\mathrm{e}^{-}$ photoproduction. Phys. Rev. Lett., 103, 170403.

29. Dumlu C. K. \& Dunne G. V. (2010). The Stokes Phenomenon and Schwinger Vacuum Pair Production in TimeDependent Laser Pulses. Phys. Rev. Lett., 104, 250402.

30. Korn G., (2011). WhiteBook on ELI Science and Technology with Ultra-Intense Lasers. CNRS: Cedex.

31. Garrec B. L., Sebban S., Margarone D., Precek M., Weber S., Klimo O., Korn G., \& Rus B. (2014). ELIbeamlines: Extreme light infrastructure science and technology with ultra-intense lasers. Proc. SPIE, 8962, 89620I.

32. Kuhn S., Dumergue M., Kahaly S., Mondal S., Csizmadia T., Farkas B., Major B., Cormier E., Kalashnikov M., Calegari F., Devetta M., Frassetto F., Poletto L., Stagira S., Vozzi C., Nisoli M., Rudawski P., Maclot S., Campi F., Wikmark H., Arnold C. L., Heyl C. M., Johnsson P., L’Huillier A., Lopez- Martens R., Haessler S., Bocoum M., Boehle F., Vernier A., Iaquaniello G., Skantzakis E., Papadakis N., Kalpouzos C., Tzallas P., Lpine F., Charalambidis D., Osvay K., \& Sansone G. J. (2017). The ELI-ALPS facility: the next generation of attosecond sources. Phys. B: At. Mol. Opt. Phys., 50, 132002.

33. Gales S., Tanaka K. A.,. Balabanski D. L, Negoita F., Stutman D., Tesileanu O., Ur C. A., Ursescu D., Andrei I., Ataman S., Cernaianu M. O., D’Alessi L., Dancus I., Diaconescu B., Djourelov N., Filipescu D., Ghenuche P., Ghita D. G., Matei C., Seto K., Zeng M., \& Zamfir N. V. (2018). The extreme light infrastructure-nuclear physics (ELI-NP) 
facility: new horizons in physics with 10 PW ultra-intense lasers and $20 \mathrm{MeV}$ brilliant gamma beams. Rep. Prog. Phys., 81, 094301.

34. Bulanov S. S., Mur V. D., Narozhny N. B., Nees J., \& Popov V. S. (2010). Multiple colliding electromagnetic pulses: a way to lower the threshold of e+e- pair production from vacuum. Phys. Rev. Lett., 104, 220404.

35. Bulanov S. S., Esirkepov T. Z., Thomas A. G. R., Koga J. K., \& Bulanov S. V. (2010). On the Schwinger limit attainability with extreme power lasers. Phys. Rev. Lett., 105, 220407.

36. Murakami M., Arefiev A., \& Zosa M. A. (2018). Generation of ultrahigh field by micro-bubble implosion. Sci. Rep., 8, 7537.

37. Arber T. D., Bennett K., Brady C. S., Lawrence-Douglas A., Ramsay M. G., Sircombe N. J., Gillies P., Evans R. G., Schmitz H., Bell A. R., \& Ridgers C. P. (2015). Contemporary particle-in-cell approach to laser-plasma modeling. Plasma Phys. Controlled Fusion, 57, 113001.

38. Murakami M., Sarukura N., Azechi H., Temporal M., \& Schmitt A. J. (2010). Optimization of irradiation configuration in laser fusion utilizing self-organizing electrodynamic system. Phys. Plasmas, 17, 082702.

39. Koga J., Murakami M., Arefiev A.V., \& Nakamiya Y. (2019). Probing and possible application of the QED vacuum with micro-bubble implosions induced by ultra-intense laser pulses. Matter Radiat. Extremes, 4, 034401. 